\title{
Ligand Effects on the Formation of Imino Complexes
}

\author{
Chung-Yuan Liu, Ming-Chu Cheng, Shie-Ming Peng, and Shiuh-Tzung Liu* \\ Department of Chemistry, National Taiwan University, Taipei, Taiwan 106, Republic of China
}

Received April 22, 1994 ${ }^{\otimes}$

\begin{abstract}
The photochemical substitution reaction of $\mathrm{CpFe}(\mathrm{CO})_{2} \mathrm{I}$ with the amino-phosphine ligands $\mathrm{CH}_{3} \mathrm{C}\left(\mathrm{CH}_{2} \mathrm{PPh}_{2}\right)_{2}\left(\mathrm{CH}_{2} \mathrm{NH}_{2}\right)\left(\mathrm{P}_{2} \mathrm{~N}\right), \mathrm{Ph}_{2} \mathrm{PCH}_{2} \mathrm{CH}\left(\mathrm{NH}_{2}\right) \mathrm{CH}_{2} \mathrm{PPh}_{2}(\mathrm{PNP}), \mathrm{Ph}_{2} \mathrm{PCH}_{2} \mathrm{CH}\left(\mathrm{PPh}_{2}\right) \mathrm{CH}_{2}-$ $\mathrm{NH}_{2}$ (PPN), $\mathrm{Ph}_{2} \mathrm{PCH}_{2} \mathrm{CH}\left(\mathrm{CH}_{3}\right) \mathrm{NH}_{2}(\mathrm{PN}-2)$, and $\mathrm{H}_{2} \mathrm{~N}\left(\mathrm{CH}_{2}\right)_{3} \mathrm{PPh}_{2}$ (PN-3) resulted in the formation of $\left[\mathrm{CpFe}\left(P, P^{\prime}, N-\mathrm{P}_{2} \mathrm{~N}\right)\right] \mathrm{I}(\mathbf{1 0}),\left[\mathrm{CpFe}\left(P, P^{\prime}, N\right.\right.$-PNP)]I (11), [CpFe $\left(P, P^{\prime}, N\right.$-PPN)]I (12), $[\mathrm{CpFe}(\mathrm{CO})(P, N$-PN-2)]I (13), and $[\mathrm{CpFe}(\mathrm{CO})(P, N-\mathrm{PN}-3)] \mathrm{I}(14)$, respectively. Complexes 10 and 12 were easily converted into their corresponding imino complexes [CpFe $\left\{P, P^{\prime}, N\right.$ $\left.\left.\left[\mathrm{CH}_{3} \mathrm{C}\left(\mathrm{CH}_{2} \mathrm{PPh}_{2}\right)_{2}(\mathrm{CH}=\mathrm{NH})\right]\right\}\right] \mathrm{I}(\mathbf{1 5})$ and $\left[\mathrm{CpFe}\left\{P, P^{\prime}, N-\left[\mathrm{Ph}_{2} \mathrm{PCH}_{2} \mathrm{CH}\left(\mathrm{PPh}_{2}\right)(\mathrm{CH}=\mathrm{NH})\right]\right\}\right] \mathrm{I}(\mathbf{1 6})$, respectively, whereas the other complexes resisted such oxidation processes. Substitution of carbonyl by trimethylphosphine in 14 resulted in conversion of the amino function into the corresponding imino group. It appears that strong $\pi$-acceptor ligands bound to the metal ion center prevent oxidation. Crystal structures of the imino complexes 15 and 16 and the amino complex 14 were determined. The reasons that 11 does not convert into its corresponding imino complex are also discussed.
\end{abstract}

\section{Introduction}

Primary amine metal complexes, being the precursors of both amido complexes by deprotonation and imino complexes by oxidation (Scheme 1), have received much attention. ${ }^{1-9}$ Oxidation of amine-metal complexes generally occurs spontaneously in air with the assistance of a metal ion, which results in the formation of stable imino complexes. Oxidation of this kind is suppressed by using an amine without an $\alpha$-hydrogen or a metal ion less readily oxidized. 6,7

An iron-amine complex is known to easily form its corresponding imino complex, due to facile interconversion of $\mathrm{Fe}$ (II) to $\mathrm{Fe}$ (III). ${ }^{6}$ To improve our understanding of the ligand effects of iron complexes in the conversion of an amine into an imine, we investigated the coordination behavior of amino-phosphine ligands 1-5 toward $\left(\eta^{5}\right.$-cyclopentadienyl)iron complexes and their properties.

\section{Experimental Section}

${ }^{1} \mathrm{H},{ }^{13} \mathrm{C}$, and ${ }^{31} \mathrm{P}$ NMR spectra were determined on a Bruker AC-E 200 or a Bruker AM-300WB spectrometer. Chemical shifts are given in parts per million relative to TMS for ${ }^{1} \mathrm{H}$ and ${ }^{13} \mathrm{C} \mathrm{NMR}$ and $85 \% \mathrm{H}_{3} \mathrm{PO}_{4}$ for ${ }^{31} \mathrm{P} \mathrm{NMR}$ spectra in $\mathrm{CDCl}_{3}$, unless otherwise noted. Infrared spectra were obtained on Perkin-Elmer 983G and Biorad FT-IR instruments. Elemental analyses were carried out on a Perkin-Elmer $240 \mathrm{C}$ instrument. Cyclic voltammetry was performed with a universal program-

Abstract published in Advance ACS Abstracts, October 1, 1994. (1) Bryndza, H. E.; Tam, W. Chem. Rev. 1988, 88, 1163.

(2) Fryzuk, M. D.; Montgomery, C. D. Coord. Chem. Rev. 1989, 95,

(3) Dabrowiak, J. C.; Busch, D. H. Inorg. Chem. 1975, 14, 1881

(4) Diamond, S. E.; Tom, G. M.; Taube, H. J. Am. Chem. Soc. 1975, $97,2661$.

(5) Castro, C. E.; Jamin, M.; Yokoyama, W.; Wade, R. J. Am. Chem. Soc. 1986, 108, 4179 .

(6) Goedken, V. L. J. Chem. Soc., Chem. Commun. 1972, 207.

(7) Joslin, F. L.; Johnson, M. P.; Mague, J. T.; Roundhill, D. M. Organometallics 1991, 10, 41 .

(8) Farnetti, E.; Nardin, G.; Graziani, M. J. Organomet. Chem. 1991, $417,163$.

(9) Liu, C.-Y.; Cheng, M.-C.; Peng, S.-M.; Liu, S.-T. J. Organomet. Chem. 1994, 468, 199.
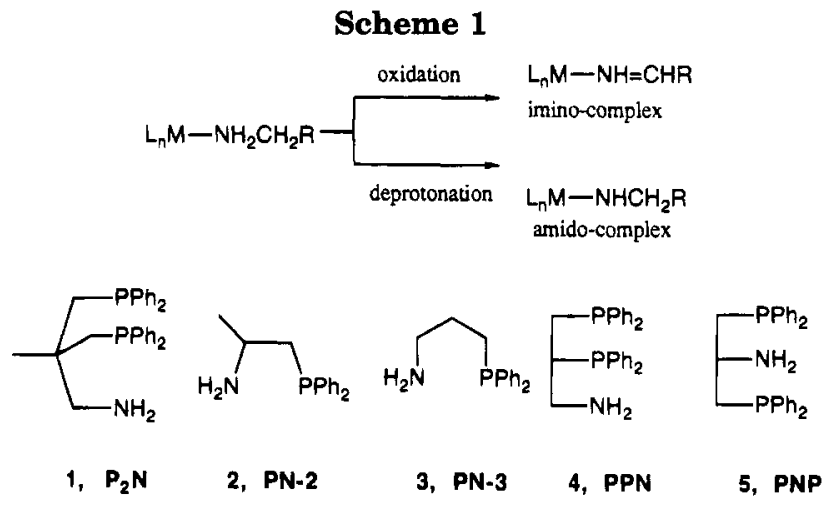

Scheme 2. Preparation of PNP and PPN Ligands
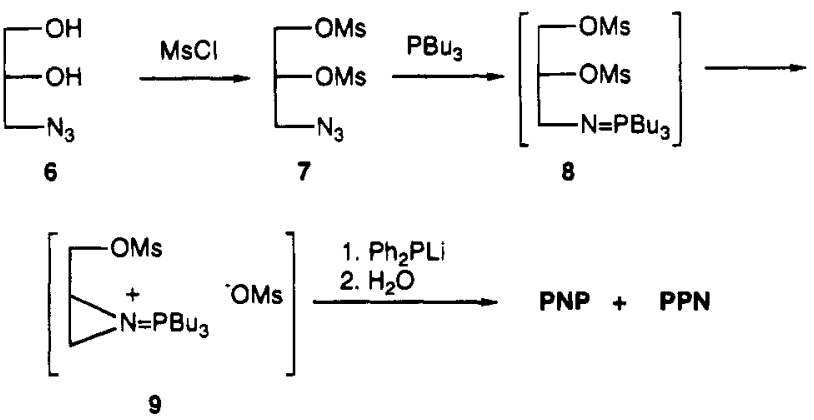

mer (Princeton Applied Research PAR-175) and a potentiostat (Model 173). Glassy carbon (Tokai) was used as a working electrode. Tetrabutylammonium perchlorate $(0.1 \mathrm{M})$ was the base electrolyte, and all data were obtained in acetonitrile solutions at $25^{\circ} \mathrm{C}$. All reactions, manipulations, and purification steps involving phosphines were performed under a dry nitrogen atmosphere.

Preparation of Ligands PNP and PPN. Both ligands were prepared according to the synthetic route shown in Scheme 2.

Methanesulfonyl chloride $(21 \mathrm{~mL}, 31.0 \mathrm{~g}, 27.1 \mathrm{mmol})$ was slowly added to a solution of compound $6^{10}(14.5 \mathrm{~g}, 12.4 \mathrm{mmol})$

(10) Gichner, T.; Holy, A.; Spassova, M.; Veleminsky, J.; Gruz, P. Mutagenesis 1991, 6, 55 . 
and triethylamine $(50 \mathrm{~mL})$ in dichloromethane $(100 \mathrm{~mL})$ at ice-water temperature. After addition, the resulting mixture was warmed to room temperature and stirred for another 2 h. Water $(50 \mathrm{~mL})$ was added to quench the reaction, and the organic layer was separated. The organic layer was washed with aqueous $\mathrm{NaHCO}_{3}$ and brine and dried with $\mathrm{MgSO}_{4}$. Concentration of the extract gave the crude product as an oily liquid which was then chromatographed on silica gel with hexane/ethyl acetate $(1 / 3 \mathrm{v} / \mathrm{v})$ as eluent. The eluate was collected and concentrated to give 7 as a clear, colorless liquid $(27.3 \mathrm{~g}, 81 \%)$ : ${ }^{1} \mathrm{H}$ NMR $\delta 4.94-4.87(\mathrm{~m}, 1 \mathrm{H},-\mathrm{CHOMs}), 4.44-$ $4.25\left(\mathrm{~m}, 2 \mathrm{H},-\mathrm{CH}_{2} \mathrm{OMs}\right), 3.70-3.57\left(\mathrm{~m}, 2 \mathrm{H},-\mathrm{CH}_{2} \mathrm{~N}_{3}\right), 3.13$ $\left(\mathrm{s}, 3 \mathrm{H},-\mathrm{CH}_{3}\right), 3.07\left(\mathrm{~s}, 3 \mathrm{H},-\mathrm{CH}_{3}\right) ;{ }^{13} \mathrm{C} \mathrm{NMR} \delta 76.2(-\mathrm{CHO}-)$, $67.3\left(-\mathrm{CH}_{2} \mathrm{O}-\right), 50.8\left(-\mathrm{CH}_{2} \mathrm{~N}_{3}\right), 38.6\left(-\mathrm{SCH}_{3}\right), 37.7\left(-\mathrm{SCH}_{3}\right)$. Anal. Calcd for $\mathrm{C}_{5} \mathrm{H}_{11} \mathrm{~S}_{2} \mathrm{~N}_{3} \mathrm{O}_{6}: \mathrm{C}, 21.98 ; \mathrm{H}, 4.06 ; \mathrm{N}, 15.38$. Found: $\mathrm{C}, 22.31 ; \mathrm{H}, 3.95 ; \mathrm{N}, 15.48$.

A solution of tributylphosphine $(7.5 \mathrm{~g}, 37.1 \mathrm{mmol})$ in THF $(100 \mathrm{~mL})$ was added slowly to a solution of compound $7(10 \mathrm{~g}$, $36.6 \mathrm{mmol})$ in THF $(200 \mathrm{~mL})$, and the reaction mixture was stirred at room temperature for 2 days. Diphenylphosphide anion was prepared by addition of a hexane solution $(1.6 \mathrm{M})$ of butyllithium to a solution of diphenylphosphine $(14.5 \mathrm{~g}, 78$ $\mathrm{mmol})$ in THF (200 $\mathrm{mL}$ ) and added to the above solution. The resulting mixture was heated to reflux for $3 \mathrm{~h}$. Water $(50 \mathrm{~mL})$ was then added, and the mixture was refluxed overnight. After the mixture was cooled to room temperature, the organic layer was separated, dried, and concentrated. The residue was chromatographed on silica gel with hexane/ethyl acetate (4/1) as eluent to give a mixture of PPN and PNP in the ratio of $1 / 4$ $(9.32 \mathrm{~g}, 60 \%)$. This mixture was rechromatographed on silica gel with ethyl acetate as eluent to separate PPN from PNP:

PNP: ${ }^{1} \mathrm{H}$ NMR $\delta 7.76-7.24(\mathrm{~m}, 20 \mathrm{H}, \mathrm{Ar} H), 2.93(\mathrm{~m}, 1 \mathrm{H}$, $-\mathrm{CHN}$ ), $2.50\left(\mathrm{ddd},{ }^{2} J=13.6 \mathrm{~Hz},{ }^{3} J_{\mathrm{H}-\mathrm{H}}=5.2 \mathrm{~Hz},{ }^{2} J_{\mathrm{P}-\mathrm{H}}=1.6\right.$ $\mathrm{Hz}, 2 \mathrm{H},-\mathrm{C} H \mathrm{HPPh}$ ), $2.19\left(\mathrm{dd},{ }^{2} J_{\mathrm{H}-\mathrm{H}}=13.6,{ }^{3} J_{\mathrm{H}-\mathrm{H}}=8.1 \mathrm{~Hz}\right.$, $\left.2 \mathrm{H},-\mathrm{CH} H \mathrm{PPh}_{2}\right), 2.15-2.05\left(\mathrm{br}, 2 \mathrm{H},-\mathrm{NH}_{2}\right) ;{ }^{13} \mathrm{C} \mathrm{NMR} \delta 138-$ 128.2 (aromatic), $47.0\left(\mathrm{t}, J_{\mathrm{P}-\mathrm{C}}=15 \mathrm{~Hz}, \mathrm{CNH}_{2}\right.$ ), 39.3 (dd, ${ }^{1} J_{\mathrm{P}-\mathrm{C}}$ $=12.7,{ }^{3} \mathrm{~J}_{\mathrm{P}-\mathrm{C}}=7.8 \mathrm{~Hz}, C \mathrm{PPh}$ ); ${ }^{31} \mathrm{P} \mathrm{NMR} \delta-22.4$. Anal. Calcd for $\mathrm{C}_{27} \mathrm{H}_{27} \mathrm{NP}_{2}$ : C, 75.87; H, 6.37; N, 3.28. Found: C, 75.65; $\mathrm{H}, 6.03 ; \mathrm{N}, 3.20$.

PPN: ${ }^{1} \mathrm{H}$ NMR $\delta 7.55-7.24(\mathrm{~m}, 20 \mathrm{H}, \mathrm{Ar} H), 3.10(\mathrm{~m}, 1 \mathrm{H}$, $\left.-\mathrm{CH} H \mathrm{NH}_{2}\right), 2.95\left(\mathrm{~m}, 1 \mathrm{H},-\mathrm{C} H \mathrm{HNH}_{2}\right), 2.33(\mathrm{~m}, 1 \mathrm{H},-\mathrm{CHP}-$ $\left.\mathrm{Ph}_{2}\right), 2.16\left(\mathrm{~m}, 2 \mathrm{H},-\mathrm{CH}_{2} \mathrm{PPh}_{2}\right), 1.40\left(\mathrm{br}, 2 \mathrm{H},-\mathrm{NH}_{2}\right){ }^{13} \mathrm{C} \mathrm{NMR}$ $\delta 138.8-128.1$ (aromatic), $42.5\left(\mathrm{dd},{ }^{2} J_{\mathrm{P}-\mathrm{C}}=14.6,{ }^{3} J_{\mathrm{P}-\mathrm{C}}=9.9\right.$ $\left.\mathrm{Hz},-\mathrm{CHNH}_{2}\right), 36.7\left(\mathrm{dd},{ }^{1} J_{\mathrm{P}-\mathrm{C}}=12.8 \mathrm{~Hz},{ }^{2} J_{\mathrm{P}-\mathrm{C}}=12.3 \mathrm{~Hz}\right.$, $\left.-C \mathrm{HPPh}_{2}\right), 28.1\left(\mathrm{dd},{ }^{1} J_{\mathrm{P}-\mathrm{C}}=15.8,{ }^{2} J_{\mathrm{P}-\mathrm{C}}=15.5 \mathrm{~Hz},-\mathrm{CH}_{2}\right.$ $\left.\mathrm{PPh}_{2}\right)$; ${ }^{11} \mathrm{P}$ NMR $\left.\delta-7.2(\mathrm{~d}, J=20 \mathrm{~Hz},-\mathrm{CHPPh})_{2}\right)-20.0$ (d, $J$ $=20 \mathrm{~Hz},-\mathrm{CH}_{2} P \mathrm{Ph}_{2}$ ); HRMS calcd for $\mathrm{C}_{27} \mathrm{H}_{27} \mathrm{NP}_{2} 427.1619$, found 427.1615 .

Preparation of Amino Complexes. A 0.1 M solution of the amine-phosphine and $\mathrm{CpFe}(\mathrm{CO})_{2} \mathrm{I}$ in equimolar proportions in benzene was photolyzed by a $450 \mathrm{~W}$ Conrad Hanovia medium-pressure mercury lamp (Ace Glass) at room temperature for $5 \mathrm{~h}$, and the desired product was precipitated. We collected the solid and recrystallized it from chloroform/ pentane. Physical and spectral data for all complexes follow.

[2,2-Bis[(diphenylphosphino)methyl]-1-aminopropane$\left.\boldsymbol{P}, \boldsymbol{P}^{\prime}, \boldsymbol{N}\right]\left(\boldsymbol{\eta}^{5}\right.$-cyclopentadienyl)iron(II) Iodide (10): brick red solid (75\%); mp $175-200{ }^{\circ} \mathrm{C} \mathrm{dec} ;{ }^{1} \mathrm{H}$ NMR $\delta 7.70-6.90(\mathrm{~m}, 20$ $\mathrm{H}, \mathrm{Ar} H$ ), 4.32 (s, $5 \mathrm{H}, \mathrm{Cp} H$ ), 3.85-3.65 (br, $2 \mathrm{H},-\mathrm{N} H_{2}$ ), 2.3 $\left(\mathrm{m}, 2 \mathrm{H},-\mathrm{CH}_{2} \mathrm{~N}\right), 1.93\left(\mathrm{~m}, 4 \mathrm{H},-\mathrm{CH}_{2} \mathrm{PPh}_{2}\right), 0.92(\mathrm{~s}, 3 \mathrm{H}$, $\left.-\mathrm{C} H_{3}\right) ;{ }^{13} \mathrm{C}$ NMR $\delta 140.2\left(\mathrm{~d},{ }^{1} J_{\mathrm{P}-\mathrm{C}}=19.5 \mathrm{~Hz}\right), 135.5-128.2$ (aromatic), $77.8(\mathrm{Cp} \mathrm{C}), 45.5\left(-\mathrm{CNH}_{2}\right), 36.1\left(\mathrm{dd},{ }^{1} J_{\mathrm{P}-\mathrm{C}}=8.7\right.$ $\left.\mathrm{Hz},{ }^{3} J_{\mathrm{P}-\mathrm{C}}=1.2 \mathrm{~Hz},-\mathrm{CPPh}_{2}\right), 35.0(-\mathrm{CMe}), 31.9\left(-\mathrm{CH}_{3}\right) ;{ }^{31} \mathrm{P}$ NMR $\delta$ 64.3. Anal. Calcd for $\mathrm{C}_{34} \mathrm{H}_{36} \mathrm{NP}_{2} \mathrm{FeI}$ : C, 58.06; $\mathrm{H}, 5.16$; $\mathrm{N}, 1.99$. Found: C, 58.28; H, 5.16; N, 1.98 .

$\left[1,3-B i s(d i p h e n y l p h o s p h i n o)-2\right.$-aminopropane- $\left.P, P^{\prime}, N\right]$ ( $\eta^{5}$-cyclopentadienyl)iron(II) Iodide (11): red solid powder (77\%); mp 160-180 ${ }^{\circ} \mathrm{C}$ dec; ${ }^{1} \mathrm{H}$ NMR $\delta 7.42-7.00(\mathrm{~m}, 20 \mathrm{H}, \mathrm{Ar}$ $H), 4.25(\mathrm{~s}, 5 \mathrm{H}, \mathrm{Cp} H), 4.05\left(\mathrm{~m}, 1 \mathrm{H},-\mathrm{CHNH}_{2}\right), 2.28(\mathrm{~m}, 4 \mathrm{H}$, $\left.-\mathrm{CH}_{2} \mathrm{PPh}_{2}\right), 1.70\left(\mathrm{br}, 2 \mathrm{H},-\mathrm{NH}_{2}\right), 7.24\left(\mathrm{CHCl}_{3}\right.$, solvent); ${ }^{13} \mathrm{C}$ NMR $\delta 140-128.3$ (aromatic), $76.1(\mathrm{Cp} C), 51.7\left(\mathrm{t},{ }^{2} J_{\mathrm{P}-\mathrm{C}}=4.8\right.$ $\left.\mathrm{Hz},-C \mathrm{NH}_{2}\right), 36.0\left(\mathrm{dd},{ }^{1} J_{\mathrm{P}-\mathrm{C}}=10.5 \mathrm{~Hz},{ }^{3} J_{\mathrm{P}-\mathrm{C}}=1.2 \mathrm{~Hz}\right.$,
$\left.-C P P h_{2}\right) ;{ }^{31} \mathrm{P}$ NMR $\delta$ 77.7. Anal. Calcd for $\mathrm{C}_{32} \mathrm{H}_{32} \mathrm{NP}_{2}$ $\mathrm{FeI} \cdot 1 / 2 \mathrm{CHCl}_{3}$ : C, 53.55; H, 4.70; N, 2.19. Found: C, 53.11; H, $4.46 ; \mathrm{N}, 2.19$.

[2,3-Bis(diphenylphosphino)-1-aminopropane-P,P,N]( $\eta^{5}$-cyclopentadienyl)iron(II) Iodide (12): red solid (87\%); ${ }^{1} \mathrm{H}$ NMR $\delta 7.98-6.60(\mathrm{~m}, 20 \mathrm{H}, \operatorname{Ar} H), 4.63(\mathrm{~s}, 5 \mathrm{H}, \mathrm{Cp} H)$, $4.25(\mathrm{~m}, 1 \mathrm{H},-\mathrm{CHPPh}), 2.81\left(\mathrm{~m}, 1 \mathrm{H},-\mathrm{CH} H \mathrm{NH}_{2}\right), 2.54(\mathrm{~m}$, $\left.1 \mathrm{H},-\mathrm{C} H \mathrm{HNH}_{2}\right), 2.38(\mathrm{br}, 1 \mathrm{H},-\mathrm{NH}), 2.18(\mathrm{~m}, 1 \mathrm{H},-\mathrm{CH} \mathrm{H}-$ $\left.\mathrm{PPh}_{2}\right), 1.93\left(\mathrm{~m}, 1 \mathrm{H},-\mathrm{CH} H \mathrm{PPh}_{2}\right), 1.69(\mathrm{br}, 1 \mathrm{H},-\mathrm{NH}) ;{ }^{31} \mathrm{P}$ NMR $\delta 102.5(\mathrm{~d}, J=26 \mathrm{~Hz},-\mathrm{CHPPh}), 84.6(\mathrm{~d}, J=26 \mathrm{~Hz}$, $\left.-\mathrm{CH}_{2} P \mathrm{Ph}_{2}\right) ; \mathrm{FAB} m / z$ for $[\mathrm{M}-\mathrm{I}] 548$.

[1-(Diphenylphosphino)-2-aminopropane- $P, N]\left(\eta^{5}\right.$-cyclopentadienyl)carbonyliron(II) Iodide (13): red solid (73\%); mp 212-220 ${ }^{\circ} \mathrm{C} \mathrm{dec;} \mathrm{IR} \mathrm{(KBr)} v_{\mathrm{CO}} 1947 \mathrm{~cm}^{-1}$; ${ }^{1} \mathrm{H}$ NMR $\delta$ 7.80-7.40 (m, $10 \mathrm{H}, \operatorname{Ar} H), 5.18(\mathrm{~m}, 1 \mathrm{H},-\mathrm{NHH}), 4.80(\mathrm{~s}, 5$ $\mathrm{H}, \mathrm{Cp} H), 3.30(\mathrm{~m}, 1 \mathrm{H},-\mathrm{NH} H), 2.78\left(\mathrm{br}, 2 \mathrm{H},-\mathrm{CH}_{2} \mathrm{PPh}_{2}\right.$ ), $2.35(\mathrm{~m}, 1 \mathrm{H},-\mathrm{CHCH})_{3}, 1.68\left(\mathrm{~d},{ }^{2} J_{\mathrm{H}-\mathrm{H}}=5.5 \mathrm{~Hz}, 3 \mathrm{H},-\mathrm{Me}\right)$; ${ }^{13} \mathrm{C}$ NMR $\delta 218.1\left(\mathrm{~d},{ }^{2} J_{\mathrm{P}-\mathrm{C}}=25.3 \mathrm{~Hz},-C \mathrm{O}\right), 135.5-128.8$ (aromatic), $82.2(\mathrm{Cp} C), 55.84\left(\mathrm{~d},{ }^{2} J_{\mathrm{P}-\mathrm{C}}=6.8 \mathrm{~Hz},-\mathrm{CN}\right), 37.3$ $\left(\mathrm{d},{ }^{1} J_{\mathrm{P}-\mathrm{C}}=24.7 \mathrm{~Hz},-\mathrm{CPPh}_{2}\right), 22.9\left(\mathrm{~d},{ }^{3} \mathrm{~J}_{\mathrm{P}-\mathrm{C}}=13.7 \mathrm{~Hz},-\mathrm{CH}_{3}\right)$; 31P NMR $\delta$ 83.0. Anal. Calcd for $\mathrm{C}_{21} \mathrm{H}_{23} \mathrm{NOPFeI}$ C, 48.59; $\mathrm{H}, 4.47 ; \mathrm{N}, 2.70$. Found: C, 48.68; H, 4.57; N, 2.36.

[1-(Diphenylphosphino)-3-aminopropane- $P, N]\left(\eta^{5}\right.$-cyclopentadienyl)carbonyliron(II) Iodide (14): red crystalline solid (85\%); mp $181-187^{\circ} \mathrm{C} \mathrm{dec}$; IR ( $\left.\mathrm{KBr}\right) v_{\mathrm{CO}} 1942 \mathrm{~cm}^{-1}$; ${ }^{1} \mathrm{H}$ NMR $\delta 8.0-7.35(\mathrm{~m}, 10 \mathrm{H}, \mathrm{Ar} H), 4.84(\mathrm{~s}, 5 \mathrm{H}, \mathrm{Cp} H), 4.15$ $(\mathrm{m}, 1 \mathrm{H},-\mathrm{NH}), 3.78(\mathrm{~m}, 1 \mathrm{H},-\mathrm{NH}), 3.25(\mathrm{~m}, 1 \mathrm{H},-\mathrm{CH} H \mathrm{~N})$, $2.50(\mathrm{~m}, 1 \mathrm{H},-\mathrm{CHHPPh}), 2.02\left(\mathrm{~m}, 1 \mathrm{H},-\mathrm{CH} H \mathrm{PPh}_{2}\right), 1.9(\mathrm{~m}$, $1 \mathrm{H},-\mathrm{CH} H-) 1.81(\mathrm{~m}, 1 \mathrm{H},-\mathrm{CHHN}), 1.60(\mathrm{~m}, 1 \mathrm{H},-\mathrm{CHH}-)$, $7.24\left(\mathrm{CHCl}_{3}\right.$, solvent); ${ }^{13} \mathrm{C} \mathrm{NMR} \delta 218.1\left(\mathrm{~d},{ }^{2} J_{\mathrm{P}-\mathrm{C}}=28.3 \mathrm{~Hz}\right.$, -CO), 134-129.0 (aromatic), $83.35(\mathrm{Cp} \mathrm{C}), 44.26\left(-\mathrm{CNH}_{2}\right.$ ), $26.8\left(\mathrm{~d}, J_{\mathrm{P}-\mathrm{C}}=24.2 \mathrm{~Hz},-\mathrm{CPPh}_{2}\right), 22.4\left(-\mathrm{CH}_{2}-\right) ;{ }^{31} \mathrm{P} \mathrm{NMR}$ 55.5. Anal. Calcd for $\mathrm{C}_{21} \mathrm{H}_{23} \mathrm{NOPFeI}$ : C, 48.59; H, 4.47; N, 2.70. Found: C, $48.20 ; \mathrm{H}, 4.56 ; \mathrm{N}, 2.45$.

[1-(Diphenylphosphino)-3-aminopropane-P,N]( $\eta^{5}$-cyclopentadienyl)(trimethylphosphine)iron(II) Iodide (18). A solution of 14 ( $145 \mathrm{mg}, 0.279 \mathrm{mmol})$ and trimethylphosphine $(21.3 \mathrm{mg}, 0.28 \mathrm{mmol})$ in acetonitrile $(30 \mathrm{~mL})$ was irradiated with light for $12 \mathrm{~h}$. Removal of solvent and recrystallization from chloroform/pentane gave a red crystalline solid as the desired product (105 mg, 66\%): $\mathrm{mp} 160-165{ }^{\circ} \mathrm{C} \mathrm{dec} ;{ }^{1} \mathrm{H}$ NMR d 7.80-7.20 (m, 10 H, Ar H), 4.22 (s, $5 \mathrm{H}, \mathrm{Cp} \mathrm{H}), 4.03(\mathrm{~m}, 1 \mathrm{H}$, $-\mathrm{NH}), 3.22(\mathrm{~m}, 1 \mathrm{H},-\mathrm{NH}), 3.03(\mathrm{~m}, 1 \mathrm{H},-\mathrm{CH} H \mathrm{~N}), 2.60(\mathrm{~m}$, $\left.1 \mathrm{H},-\mathrm{CHHPPh} \mathrm{H}_{2}\right), 2.25\left(\mathrm{~m}, 1 \mathrm{H},-\mathrm{CH} H \mathrm{PPh}_{2}\right), 2.03(\mathrm{~m}, 1 \mathrm{H}$, $-\mathrm{CH} H \mathrm{~N}), 1.58\left(\mathrm{~m}, 2 \mathrm{H},-\mathrm{CH}_{2}-\right), 1.34\left(\mathrm{~d},{ }^{2} J_{\mathrm{P}-\mathrm{H}}=7.3 \mathrm{~Hz}, 9 \mathrm{H}\right.$, $\left.-\mathrm{P}\left(\mathrm{CH}_{3}\right)_{3}\right) ;{ }^{13} \mathrm{C} \mathrm{NMR} \delta 134.2-128.6$ (aromatic), $77.2(\mathrm{Cp} \mathrm{C})$, $41.7\left(-\mathrm{CNH}_{2}\right), 24.9\left(\mathrm{~d},{ }^{1} \mathrm{~J}_{\mathrm{P}-\mathrm{C}}=18 \mathrm{~Hz},-\mathrm{CPPh}_{2}\right), 23.1\left(-\mathrm{CH}_{2}-\right)$ $20.9\left(\mathrm{~d},{ }^{1} J_{\mathrm{P}-\mathrm{C}}=23 \mathrm{~Hz}, \mathrm{P}-\mathrm{CH}_{3}\right) ;{ }^{31} \mathrm{P}$ NMR $\delta 57.8(\mathrm{~d}, J=53$ $\left.\mathrm{Hz},-P \mathrm{Ph}_{2}\right), 19.4\left(\mathrm{~d}, J=53 \mathrm{~Hz},-P \mathrm{Me}_{3}\right) ; \mathrm{FAB} m / z$ for $[\mathrm{M}-\mathrm{I}]$ 440 .

Dicarbonyl[1-(Diphenylphosphino)-3-aminopropane$P]\left(\eta^{5}\right.$-cyclopentadienyl)iron(II) Iodide (17). The ligand and $\mathrm{CpFe}(\mathrm{CO})_{2} \mathrm{I}$ in equimolar proportions in benzene solution were stirred for $10 \mathrm{~h}$ at room temperature. A yellow solid formed, was filtered, and was identified as 17: yellow solid (92\%) (decomposed slowly in air); IR (KBr) $\nu_{\mathrm{CO}} 2047,2001$ $\mathrm{cm}^{-1} ;{ }^{1} \mathrm{H}$ NMR $\left(\mathrm{CD}_{3} \mathrm{CN}\right) \delta 7.75-7.45(\mathrm{~m}, 10 \mathrm{H}, \mathrm{Ar} H), 5.33(\mathrm{~s}$, $5 \mathrm{H}, \mathrm{Cp} H), 2.83\left(\mathrm{~m}, 2 \mathrm{H},-\mathrm{CH}_{2} \mathrm{P}\right), 2.65-2.50(\mathrm{~m}, 6 \mathrm{H}$, $-\left(\mathrm{CH}_{2}\right)_{2} \mathrm{~N}-$ and $\left.-\mathrm{NH}_{2}\right) ;{ }^{13} \mathrm{C} \mathrm{NMR}\left(\mathrm{CD}_{3} \mathrm{CN}\right) \delta 210.4\left(\mathrm{~d},{ }^{2} J_{\mathrm{P}-\mathrm{C}}\right.$ $=24 \mathrm{~Hz},-\mathrm{CO}$ ), 132.6-129.8 (aromatic), 89.0 (Cp C), 41.3 $(-\mathrm{CN}), 30.5\left(\mathrm{~d},{ }^{1} J_{\mathrm{P}-\mathrm{C}}=32.7 \mathrm{~Hz},-C \mathrm{PPh}\right), 25.8\left(-\mathrm{CH}_{2}-\right) ;{ }^{11} \mathrm{P}$ $\mathrm{NMR}\left(\mathrm{CD}_{3} \mathrm{CN}\right) \delta 60.2 ; \mathrm{FAB} \mathrm{m} / \mathrm{z}$ for $[\mathrm{M}-\mathrm{I}] 420$.

Preparation of Imino Complexes. A chloroform solution of the amine-phosphine complex was stirred in air for 4-7 h to give the desired imino complex after concentration. Recrystallization from chloroform/pentane provided a pure product.

[2,2-Bis[(diphenylphosphino)methyl]-1-propanimine$\left.\boldsymbol{P}, \boldsymbol{P}^{\prime}, \boldsymbol{N}\right]\left(\boldsymbol{\eta}^{5}\right.$-cyclopentadienyl)iron(II) Iodide (15): red solid (100\%); mp 160-210 ${ }^{\circ} \mathrm{C} \mathrm{dec;}{ }^{1} \mathrm{H}$ NMR $\delta 13.85$ (d, ${ }^{3} J=11.4$ $\mathrm{Hz}, 1 \mathrm{H},-\mathrm{C}=\mathrm{N} H), 8.43\left(\mathrm{dt},{ }^{3} J=11.4 \mathrm{~Hz},{ }^{4} J_{\mathrm{P}-\mathrm{H}}=3.9 \mathrm{~Hz}, 1\right.$ $\mathrm{H},-\mathrm{CH}=\mathrm{NH}), 7.37-6.90(\mathrm{~m}, 20 \mathrm{H}, \mathrm{Ar} H), 4.58(\mathrm{~s}, 5 \mathrm{H}, \mathrm{Cp} H)$, 
$2.05\left(\mathrm{~m}, 2 \mathrm{H},-\mathrm{CH} H \mathrm{PPh}_{2}\right), 1.70\left(\mathrm{~m}, 2 \mathrm{H},-\mathrm{CHHPPh} \mathrm{h}_{2}\right), 1.48(\mathrm{~s}$, $\left.3 \mathrm{H},-\mathrm{CH}_{3}\right), 7.24\left(\mathrm{CHCl}\right.$, solvent); ${ }^{13} \mathrm{C} \mathrm{NMR} \delta 181.3\left(\mathrm{t},{ }^{3} \mathrm{~J}_{\mathrm{P}-\mathrm{C}}\right.$ $=5 \mathrm{~Hz},-\mathrm{C}=\mathrm{N}), 139.6\left(\mathrm{~d},{ }^{1} J_{\mathrm{P}-\mathrm{C}}=21.8 \mathrm{~Hz}\right), 133.6-128.5$ (aromatic), $80.6(\mathrm{Cp} \mathrm{C}), 39.8\left(-\mathrm{CCH}_{3}\right), 33.9\left(\mathrm{t},{ }^{1} J_{\mathrm{P}-\mathrm{C}}=15.8\right.$ $\left.\mathrm{Hz},-\mathrm{CPPh}_{2}\right), 29.8\left(\mathrm{t},{ }^{3} \mathrm{~J}_{\mathrm{P}-\mathrm{C}}=10.1 \mathrm{~Hz},-\mathrm{CH}_{3}\right) ;{ }^{31} \mathrm{P} \mathrm{NMR} \delta 68.8$. Anal. Calcd for $\mathrm{C}_{34} \mathrm{H}_{34} \mathrm{NP}_{2} \mathrm{FeI} \cdot \mathrm{CHCl}_{3}$ : C, 51.22; H, 4.30; N, 1.71. Found: C, 50.95; H, 4.32; N, 1.84 .

[2,3-Bis (diphenylphosphino)-1-propanimine-P, $\left.P^{\prime}, N\right]$ ( $\eta^{5}$-cyclopentadienyl)iron(II) Iodide (16): orange solid (100\%); mp 185-200 ${ }^{\circ} \mathrm{C} \mathrm{dec;}{ }^{1} \mathrm{H}$ NMR $\delta 13.11\left(\mathrm{~d},{ }^{3} J=9.3 \mathrm{~Hz}\right.$, $1 \mathrm{H},-\mathrm{CH}=\mathrm{NH}), 7.69(\mathrm{~m}, 1 \mathrm{H},-\mathrm{CH}=\mathrm{NH}), 7.60-7.25(\mathrm{~m}, 16$ $\mathrm{H}, \operatorname{Ar} H), 7.16(\mathrm{~m}, 2 \mathrm{H}, \operatorname{Ar} H), 6.78(\mathrm{~m}, 2 \mathrm{H}, \operatorname{Ar} H), 4.74(\mathrm{~s}, 5$ $\mathrm{H}, \mathrm{Cp} H), 4.26\left(\mathrm{~m}, 1 \mathrm{H},-\mathrm{CHPPh} \mathrm{P}_{2}\right), 2.45\left(\mathrm{~m}, 1 \mathrm{H},-\mathrm{C} H \mathrm{HPPh}_{2}\right)$, $1.88\left(\mathrm{~m}, 1 \mathrm{H},-\mathrm{CH} H \mathrm{PPh}_{2}\right), 7.24\left(\mathrm{CHCl}_{3}\right.$, solvent); ${ }^{13} \mathrm{C} \mathrm{NMR} \delta$ $173.3\left(\mathrm{t},{ }^{3} J_{\mathrm{p}-\mathrm{C}}=4.7 \mathrm{~Hz},-C=\mathrm{N}\right.$ ), 135.7-128.2 (aromatic), 77.2 $(\mathrm{Cp} C), 48.3\left(\mathrm{dd},{ }^{1} J_{\mathrm{P}-\mathrm{C}}=29,{ }^{2} J_{\mathrm{P}-\mathrm{C}}=9.5 \mathrm{~Hz},-\mathrm{CHPPh}_{2}\right), 24.56$ $\left(\mathrm{dd},{ }^{1} J_{\mathrm{P}-\mathrm{C}}=32,{ }^{2} J_{\mathrm{P}-\mathrm{C}}=14 \mathrm{~Hz},-\mathrm{CH}_{2} \mathrm{PPh}_{2}\right) ;{ }^{31} \mathrm{P} \mathrm{NMR} \delta 137.5$ (d, $\left.J=20 \mathrm{~Hz},-\mathrm{CH} P \mathrm{Ph}_{2}\right), 82.1\left(\mathrm{~d}, J=20 \mathrm{~Hz},-\mathrm{CH}_{2} P \mathrm{Ph}_{2}\right)$. Anal. Calcd for $\mathrm{C}_{32} \mathrm{H}_{30} \mathrm{NP}_{2} \mathrm{FeI} \cdot 0.25 \mathrm{H}_{2} \mathrm{O}: \mathrm{C}, 55.30 ; \mathrm{H}, 4.32 ; \mathrm{N}$, 2.23. Found: 55.09; H, 4,34; N, 1.99 .

[3-(Diphenylphosphino)-1-propanimine- $P, N]\left(\eta^{5}\right.$-cyclopentadienyl)(trimethylphosphine)iron(II) Iodide (19): red solid (62\%); $\mathrm{mp} 165-175{ }^{\circ} \mathrm{C} \mathrm{dec} ;{ }^{1} \mathrm{H}$ NMR $\delta 12.77$ (d, $J=$ $12.7 \mathrm{~Hz}, 1 \mathrm{H},-\mathrm{CH}=\mathrm{NH}), 8.69(\mathrm{~m}, 1 \mathrm{H},-\mathrm{CH}=\mathrm{NH}), 7.74-7.40$ $(\mathrm{m}, 10 \mathrm{H}, \operatorname{Ar} H), 4.37(\mathrm{~s}, 5 \mathrm{H}, \mathrm{Cp} \mathrm{H}), 2.85\left(\mathrm{~m}, 2 \mathrm{H},-\mathrm{CH}_{2} \mathrm{C}=\mathrm{N}\right)$, $2.20\left(\mathrm{~m}, 1 \mathrm{H},-\mathrm{CH} H \mathrm{PPh} \mathrm{P}_{2}\right), 1.60\left(\mathrm{~m}, 1 \mathrm{H},-\mathrm{CH} \mathrm{HPPh}_{2}\right), 1.20$ $\left(\mathrm{d}, J_{\mathrm{P}-\mathrm{H}}=8.6 \mathrm{~Hz}, 9 \mathrm{H}, \mathrm{P}\left(\mathrm{CH}_{3}\right)_{3}\right) ;{ }^{13} \mathrm{C} \mathrm{NMR} \delta 181.1\left(\mathrm{dd},{ }^{3} J_{\mathrm{P}-\mathrm{C}}\right.$ $=8 \mathrm{~Hz},{ }^{3} J_{\mathrm{P}-\mathrm{C}}=3 \mathrm{~Hz},-\mathrm{C}=\mathrm{N}$ ), 134.9-128.5 (aromatic), 78.7 $(\mathrm{Cp} C), 23.5\left(\mathrm{CH}_{2} \mathrm{C}=\mathrm{N}\right), 21.5\left(\mathrm{~d}, J_{\mathrm{P}-\mathrm{C}}=18 \mathrm{~Hz}, \mathrm{CPPh}_{2}\right), 19.2$ $\left(\mathrm{d}, J_{\mathrm{P}-\mathrm{C}}=25 \mathrm{~Hz}, \mathrm{PCH}_{3}\right) ;{ }^{31} \mathrm{P} \mathrm{NMR} \delta 63.0(\mathrm{~d}, J=53 \mathrm{~Hz}$, $-P \mathrm{Ph}_{2}$ ), $24.3\left(\mathrm{~d}, J=53 \mathrm{~Hz},-P \mathrm{Me}_{3}\right.$ ); FAB mass $m / z$ for $[\mathrm{M}-$ I] 438 .

X-ray Crystallography. Single crystals suitable for X-ray analysis of complexes $14 \cdot \mathrm{CHCl}_{3}, 15 \cdot \mathrm{H}_{2} \mathrm{O} \cdot \mathrm{CHCl}_{3}$, and $16 \cdot \mathrm{H}_{2} \mathrm{O} \cdot \mathrm{CH}$ $\mathrm{Cl}_{3}$ were obtained in each case by slow evaporation of a chloroform/pentane solution under air. Cell parameters were determined on a CAD-4 diffractometer at $298 \mathrm{~K}$ by a leastsquares treatment. Atomic scattering factors were taken from ref 11. Crystal data for these complexes are summarized in Table 1, and their non-hydrogen atomic coordinates are listed in Tables $2-4$, respectively.

\section{Results and Discussion}

Preparation of Ligands. Tripodal and bidentate ligands 1-3 were obtained by our previously published procedures, ${ }^{13}$ whereas the PPN and PNP ligands were prepared according to Scheme 2 . The transformation of azido diol 6 into mesylate 7 proceeded by a standard procedure. Conversion of 7 to a mixture of PPN and PNP was accomplished in a one-pot reaction according to the following sequence: formation of iminophosphorane 8 , substitution reaction with a lithium diphenylphosphide and removal of the iminophosphorane group. The production of a mixture of PPN and PNP is due to the formation of aziridine 9 via nucleophilic displacement of iminophosphorane at the adjacent carbon and a subsequent ring opening by phosphide at both carbon centers; the ratio PPN/PNP is ca. $1 / 4$, indicating that attack at the less substituted position is much preferred.

Complexation. Photochemical substitution of $\mathrm{CpFe}$ $(\mathrm{CO})_{2} \mathrm{I}$ by the tripodal amino-phosphine ligand $\mathrm{P}_{2} \mathrm{~N}$ in benzene solution led to the precipitation of the corresponding $\left[\left(P, P^{\prime}, N-\mathrm{P}_{2} \mathrm{~N}\right) \mathrm{FeCp}\right] \mathrm{I}(\mathbf{1 0})$. PNP and PPN ligands behave similarly; the reactions give the cor-

(11) International Tables for X-ray Crystallography; Kynoch Press: Birmingham, U.K, 1974; Vol. VI.

(12) Gabe, E. J.; Lee, F. L. Acta Crystallogr. 1981, A37, S339.

(13) Liu, S.-T.; Liu, C.-Y. J. Org. Chem. 1992, 57, 6079.
Table 1. Crystal Data and Summary of Intensity Data Collection and Structure Refinement

\begin{tabular}{|c|c|c|c|}
\hline & $14 \cdot \mathrm{CHCl}_{3}$ & $15 \cdot \mathrm{CHCl}_{3} \cdot \mathrm{H}_{2} \mathrm{O}$ & $16 \cdot \mathrm{CHCl}_{3} \cdot \mathrm{H}_{2} \mathrm{O}$ \\
\hline formula & $\begin{array}{r}\mathrm{C}_{22} \mathrm{H}_{24} \mathrm{Cl}_{3-} \\
\mathrm{INOPFe}\end{array}$ & $\begin{array}{l}\mathrm{C}_{35} \mathrm{H}_{37} \mathrm{Cl}_{3^{-}} \\
\quad \mathrm{INOP} \mathrm{P}_{2} \mathrm{Fe}\end{array}$ & $\begin{array}{l}\mathrm{C}_{33} \mathrm{H}_{33} \mathrm{Cl}_{3-}- \\
\text { INOP }{ }_{2} \mathrm{Fe}\end{array}$ \\
\hline fw & 669.72 & 838.96 & 810.90 \\
\hline cryst syst & monoclinic & monoclinic & monoclinic \\
\hline space group & $P 2_{1} / n$ & $P 2_{1} / c$ & $P 2{ }_{1} / c$ \\
\hline temp/K & 298 & 298 & 298 \\
\hline$a, \AA$ & $10.959(2)$ & $10.041(2)$ & $14.940(3)$ \\
\hline$b, \AA$ & $8.111(2)$ & $15.823(3)$ & $9.120(3)$ \\
\hline$c, \AA$ & $29.247(5)$ & $22.807(4)$ & $26.340(5)$ \\
\hline$\beta, \operatorname{deg}$ & $93.30(2)$ & $92.93(2)$ & $103.99(2)$ \\
\hline$V, \AA^{3}$ & $2596(1)$ & $3619(1)$ & $3482(1)$ \\
\hline $2 \theta$ range, deg & $18.64-24.00$ & $18.74-22.66$ & $19.08-21.20$ \\
\hline$F(000)$ & 1324 & 1688 & 1624 \\
\hline$Z$ & 4 & 4 & 4 \\
\hline$D_{\text {calcd, } \mathrm{g} \mathrm{cm}^{-3}}$ & 1.714 & 1.540 & 1.547 \\
\hline$\mu, \mathrm{mm}^{-1}$ & 1.83 & 1.37 & 1.42 \\
\hline scan type & $\omega-2 \theta$ & $\omega-2 \theta$ & $\omega-2 \theta$ \\
\hline radiation & Mo $\mathrm{K} \alpha$ & Mo K $\alpha$ & Mo $\mathrm{K} \alpha$ \\
\hline cryst dimens, $\mathrm{mm}^{3}$ & $\begin{array}{c}0.20 \times 0.30 \\
\times 0.30\end{array}$ & $\begin{array}{c}0.40 \times 0.30 \\
\times 0.30\end{array}$ & $\begin{array}{c}0.30 \times 0.40 \\
\times 0.45\end{array}$ \\
\hline scan width & $0.6+0.35 \tan \theta$ & $0.8+0.35 \tan \theta$ & $0.7+0.35 \tan \theta$ \\
\hline transmissn range & $0.71-1.00$ & $0.81-1.00$ & $0.93-1.00$ \\
\hline no. of rflns measd & 4547 & 5011 & 4536 \\
\hline no. of $2 \theta \max , \operatorname{deg}$ & 50 & 45 & 45 \\
\hline no. of unique, rflns & 4547 & 4694 & 4536 \\
\hline no. of rflns obsd ${ }^{a}$ & 2685 & 3133 & 3266 \\
\hline computation & NRCSDP-VAX $^{b}$ & NRCSDP-VAX & NRCSDP-VAX \\
\hline no. of params & 272 & 392 & 380 \\
\hline$R$ & 0.042 & 0.061 & 0.056 \\
\hline$R_{\mathrm{w}}$ & 0.031 & 0.047 & 0.046 \\
\hline$S$ & 1.81 & 3.76 & 3.70 \\
\hline
\end{tabular}

${ }^{a} I>2 \sigma(I) \cdot{ }^{b}$ Reference 12.

Table 2. Atomic Coordinates and Thermal Parameters for $14 \cdot \mathrm{CHCl}_{3}$

\begin{tabular}{|c|c|c|c|c|}
\hline & $x$ & $y$ & $z$ & $B_{\mathrm{eq}}, \AA^{2}$ \\
\hline I & $0.46744(5)$ & $0.1392(7)$ & $0.087426(20)$ & $4.51(3)$ \\
\hline $\mathrm{Fe}$ & $0.77875(9)$ & $0.75687(13)$ & $0.04283(4)$ & $3.20(5)$ \\
\hline P1 & $0.79516(16)$ & $0.6435(3)$ & $0.11082(7)$ & $3.24(9)$ \\
\hline $\mathbf{N}$ & $0.5936(5)$ & $0.7636(8)$ & $0.03964(19)$ & $3.7(3)$ \\
\hline $\mathrm{Cl}$ & $0.6588(6)$ & $0.5261(9)$ & $0.1239(3)$ & $3.8(4)$ \\
\hline $\mathrm{C} 2$ & $0.5376(6)$ & $0.6104(10)$ & $0.10909(25)$ & $4.1(4)$ \\
\hline C3 & $0.5176(6)$ & $0.6296(10)$ & $0.0584(3)$ & $4.1(4)$ \\
\hline $\mathrm{C} 4$ & $0.7871(6)$ & $0.5657(9)$ & $0.01768(24)$ & $3.5(4)$ \\
\hline 04 & $0.7964(5)$ & $0.4380(6)$ & $0.00058(18)$ & $5.6(3)$ \\
\hline C6 & $0.9268(6)$ & $0.9084(9)$ & $0.0593(3)$ & $4.2(4)$ \\
\hline $\mathrm{C} 7$ & $0.8225(7)$ & $1.0061(9)$ & $0.0568(3)$ & $4.4(4)$ \\
\hline $\mathrm{C} 8$ & $0.7693(7)$ & $0.9932(9)$ & $0.0119(3)$ & $4.4(4)$ \\
\hline $\mathrm{C} 9$ & $0.8376(7)$ & $0.8901(10)$ & $-0.01323(25)$ & $4.7(4)$ \\
\hline $\mathrm{Cl0}$ & $0.9387(6)$ & $0.8349(10)$ & $0.0162(3)$ & $4.8(4)$ \\
\hline C11A & $0.9195(6)$ & $0.4938(9)$ & $0.11960(23)$ & $3.5(4)$ \\
\hline $\mathrm{C} 12 \mathrm{~A}$ & $1.0303(7)$ & $0.5244(10)$ & $0.1008(3)$ & $5.3(5)$ \\
\hline C13A & $1.1271(7)$ & $0.4138(11)$ & $0.1080(3)$ & $5.6(5)$ \\
\hline $\mathrm{C} 14 \mathrm{~A}$ & $1.1121(7)$ & $0.2747(11)$ & $0.1327(3)$ & $5.5(5)$ \\
\hline C15A & $1.0030(8)$ & $0.2410(10)$ & $0.1506(3)$ & $5.4(5)$ \\
\hline C16A & $0.9070(7)$ & $0.3510(10)$ & $0.1443(3)$ & $4.8(4)$ \\
\hline C11B & $0.8176(6)$ & $0.7817(9)$ & $0.15990(23)$ & $3.2(3)$ \\
\hline C12B & $0.9113(7)$ & $0.7593(10)$ & $0.19323(24)$ & $4.2(4)$ \\
\hline C13B & $0.9206(7)$ & $0.8632(12)$ & $0.2309(3)$ & $5.9(5)$ \\
\hline C14B & $0.8370(8)$ & $0.9854(11)$ & $0.2358(3)$ & $6.3(5)$ \\
\hline C15B & $0.7445(7)$ & $1.0104(10)$ & $0.2032(3)$ & $5.0(4)$ \\
\hline $\mathrm{C} 16 \mathrm{~B}$ & $0.7364(6)$ & $0.9090(10)$ & $0.1652(3)$ & $4.1(4)$ \\
\hline C & $0.3510(7)$ & $0.9240(12)$ & $0.1965(3)$ & $6.9(6)$ \\
\hline $\mathrm{Cll}$ & $0.4549(3)$ & $0.8026(5)$ & $0.22654(12)$ & $11.73(24)$ \\
\hline $\mathrm{Cl} 2$ & $0.2739(3)$ & $1.0491(5)$ & $0.23159(11)$ & $11.54(23)$ \\
\hline $\mathrm{Cl} 3$ & $0.2465(3)$ & $0.7984(4)$ & $0.16443(11)$ & $11.01(22)$ \\
\hline
\end{tabular}

responding amine complexes $\left[\left(P, P^{\prime}, N\right.\right.$-PNP $\left.) \mathrm{FeCp}\right] \mathrm{I}(11)$ and $\left[\left(P, P^{\prime}, N-\mathrm{PPN}\right) \mathrm{FeCp}\right] \mathrm{I}(\mathbf{1 2})$, respectively. As for the bidentates 2 and 3 , substitution reactions of the iron complex by these ligands yielded $[(P, N-2) \mathrm{FeCp}(\mathrm{CO})] \mathrm{I}$ (13) and $[(P, N-3) \mathrm{FeCp}(\mathrm{CO})] \mathrm{I}$ (14), respectively. The reaction of 3 with $\mathrm{CpFe}(\mathrm{CO})_{2} \mathrm{I}$ in benzene under thermal conditions provided the monosubstituted product $[(P$ - 
Table 3. Atomic Coordinates and Thermal Parameters for $15 \cdot \mathrm{H}_{2} \mathrm{O} \cdot \mathrm{CHCl}_{3}$

\begin{tabular}{|c|c|c|c|c|}
\hline & $x$ & $y$ & $z$ & $B_{\mathrm{eq}}, \AA^{2}$ \\
\hline I & $0.40557(8)$ & $0.61556(6)$ & $0.16530(4)$ & $7.15(5)$ \\
\hline $\mathrm{Fe}$ & $0.79966(14)$ & $0.80219(9)$ & $0.20638(6)$ & $3.35(7)$ \\
\hline P1 & $0.9533(3)$ & $0.79608(18)$ & $0.14219(11)$ & $3.13(13)$ \\
\hline $\mathrm{P} 2$ & $0.9195(3)$ & $0.72467(18)$ & $0.26897(12)$ & $3.35(13)$ \\
\hline $\mathbf{N}$ & $0.7321(8)$ & $0.6996(5)$ & $0.1720(3)$ & $4.1(4)$ \\
\hline $\mathrm{C} 1$ & $1.0166(9)$ & $0.6875(6)$ & $0.1360(4)$ & $3.5(5)$ \\
\hline $\mathrm{C} 2$ & $0.9401(9)$ & $0.6190(6)$ & $0.1685(4)$ & $3.3(5)$ \\
\hline C3 & $0.7920(10)$ & $0.6294(7)$ & $0.1575(4)$ & $4.5(6)$ \\
\hline $\mathrm{C} 4$ & $0.9666(10)$ & $0.6231(6)$ & $0.2353(4)$ & $4.2(6)$ \\
\hline $\mathrm{C5}$ & $0.9836(10)$ & $0.5323(6)$ & $0.1474(4)$ & $4.7(6)$ \\
\hline C6 & $0.7912(10)$ & $0.9195(6)$ & $0.2487(4)$ & $4.4(6)$ \\
\hline $\mathrm{C} 7$ & $0.7683(10)$ & $0.9289(6)$ & $0.1886(4)$ & $4.7(6)$ \\
\hline $\mathrm{C} 8$ & $0.6551(10)$ & $0.8853(7)$ & $0.1697(5)$ & $5.7(6)$ \\
\hline $\mathrm{C} 9$ & $0.6063(10)$ & $0.8449(7)$ & $0.2184(5)$ & $5.9(7)$ \\
\hline $\mathrm{C} 10$ & $0.6898(11)$ & $0.8640(7)$ & $0.2675(4)$ & $5.3(6)$ \\
\hline $\mathrm{C} 11 \mathrm{~A}$ & $1.1054(9)$ & $0.8612(6)$ & $0.1480(4)$ & $3.1(5)$ \\
\hline $\mathrm{C} 12 \mathrm{~A}$ & $1.1220(10)$ & $0.9231(6)$ & $0.1900(4)$ & $4.2(5)$ \\
\hline C13A & $1.2348(11)$ & $0.9722(7)$ & $0.1924(5)$ & $5.1(6)$ \\
\hline $\mathrm{Cl} 4 \mathrm{~A}$ & $1.3296(10)$ & $0.9622(7)$ & $0.1530(5)$ & $5.2(6)$ \\
\hline C15A & $1.3137(10)$ & $0.9019(8)$ & $0.1106(4)$ & $5.2(7)$ \\
\hline $\mathrm{C} 16 \mathrm{~A}$ & $1.2009(10)$ & $0.8510(7)$ & $0.1075(4)$ & $4.2(6)$ \\
\hline $\mathrm{C} 11 \mathrm{~B}$ & $0.8872(9)$ & $0.8220(6)$ & $0.0675(4)$ & $3.2(5)$ \\
\hline C12B & $0.8978(10)$ & $0.9026(6)$ & $0.0448(4)$ & $4.4(6)$ \\
\hline C13B & $0.8386(11)$ & $0.9232(7)$ & $-0.0092(4)$ & $5.8(7)$ \\
\hline $\mathrm{C} 14 \mathrm{~B}$ & $0.7753(12)$ & $0.8660(8)$ & $-0.0427(5)$ & $6.7(7)$ \\
\hline C15B & $0.7607(13)$ & $0.7871(8)$ & $-0.0220(5)$ & $7.8(8)$ \\
\hline $\mathrm{C} 16 \mathrm{~B}$ & $0.8189(13)$ & $0.7663(7)$ & $0.0335(5)$ & $6.8(8)$ \\
\hline $\mathrm{C} 21 \mathrm{~A}$ & $1.0737(10)$ & $0.7673(6)$ & $0.3014(4)$ & $4.0(5)$ \\
\hline $\mathrm{C} 22 \mathrm{~A}$ & $1.0677(12)$ & $0.8373(7)$ & $0.3375(5)$ & $5.8(7)$ \\
\hline $\mathrm{C} 23 \mathrm{~A}$ & $1.1829(13)$ & $0.8745(8)$ & $0.3606(5)$ & $8.2(8)$ \\
\hline $\mathrm{C} 24 \mathrm{~A}$ & $1.3042(12)$ & $0.8423(8)$ & $0.3473(6)$ & $9.1(9)$ \\
\hline $\mathrm{C} 25 \mathrm{~A}$ & $1.3118(11)$ & $0.7742(9)$ & $0.3113(6)$ & $8.6(9)$ \\
\hline $\mathrm{C} 26 \mathrm{~A}$ & $1.1999(11)$ & $0.7390(7)$ & $0.2879(5)$ & $5.8(7)$ \\
\hline $\mathrm{C} 21 \mathrm{~B}$ & $0.8354(10)$ & $0.6847(6)$ & $0.3341(4)$ & $3.9(5)$ \\
\hline $\mathrm{C} 22 \mathrm{~B}$ & $0.6990(10)$ & $0.6680(6)$ & $0.3278(4)$ & $4.9(6)$ \\
\hline $\mathrm{C} 23 \mathrm{~B}$ & $0.6299(12)$ & $0.6369(7)$ & $0.3742(5)$ & $7.3(8)$ \\
\hline $\mathrm{C} 24 \mathrm{~B}$ & $0.7005(14)$ & $0.6262(8)$ & $0.4269(5)$ & $8.5(9)$ \\
\hline $\mathrm{C} 25 \mathrm{~B}$ & $0.8344(15)$ & $0.6373(9)$ & $0.4326(5)$ & $9.3(9)$ \\
\hline $\mathrm{C} 26 \mathrm{~B}$ & $0.9003(12)$ & $0.6674(8)$ & $0.3857(4)$ & $6.5(7)$ \\
\hline C & $0.2823(16)$ & $0.5922(12)$ & $0.0037(6)$ & $14.4(13)$ \\
\hline $\mathrm{Cll}$ & $0.1528(7)$ & $0.6542(3)$ & $-0.02402(22)$ & $17.2(5)$ \\
\hline $\mathrm{Cl} 2$ & $0.1922(7)$ & $0.4914(5)$ & $0.00782(24)$ & $20.5(6)$ \\
\hline $\mathrm{Cl} 3$ & $0.3786(6)$ & $0.5464(6)$ & $-0.0516(3)$ & $24.1(7)$ \\
\hline $\mathrm{O}$ & $0.4322(17)$ & $0.6805(11)$ & $0.9987(8)$ & $27.4(8)$ \\
\hline
\end{tabular}

3) $\left.\mathrm{FeCp}(\mathrm{CO})_{2}\right] \mathrm{I}(\mathbf{1 7})$, which was subsequently converted into 14 by photolysis.

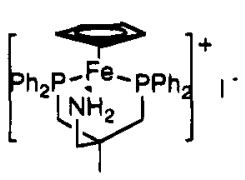

10
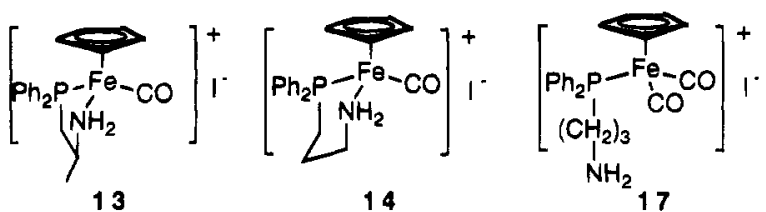

The ${ }^{31} \mathrm{P}$ NMR coordination chemical shifts of these iron complexes (Table 5) in the range 80-110 ppm are a clear indication of phosphorus donors in all ligands coordinating to the metal center. The coordination shifts of those complexes containing five-membered chelate rings appear downfield from those containing only six-membered rings, which is consistent with a ring contribution effect. ${ }^{14}$ Analogous to the coordination
Table 4. Atomic Coordinates and Thermal Parameters of $16 \cdot \mathrm{H}_{2} \mathrm{O} \cdot \mathrm{CHCl}_{3}$

\begin{tabular}{|c|c|c|c|c|}
\hline & $x$ & $y$ & $z$ & $B_{\mathrm{eq}}, \AA^{2}$ \\
\hline I & $0.21026(6)$ & $0.11711(8)$ & $0.06997(3)$ & $6.56(4)$ \\
\hline $\mathrm{Fe}$ & $0.22179(8)$ & $0.44256(14)$ & $0.92865(5)$ & $3.69(6)$ \\
\hline P1 & $0.28182(16)$ & $0.6457(3)$ & $0.96742(9)$ & $4.27(13)$ \\
\hline P2 & $0.09478(15)$ & $0.5668(3)$ & $0.89885(8)$ & $3.51(11)$ \\
\hline $\mathrm{N}$ & $0.1666(4)$ & $0.4288(7)$ & $0.98750(23)$ & $3.6(3)$ \\
\hline $\mathrm{C} 1$ & $0.1814(6)$ & $0.7576(9)$ & $0.9743(3)$ & $4.3(5)$ \\
\hline $\mathrm{C} 2$ & $0.0967(5)$ & $0.6588(9)$ & $0.9623(3)$ & $3.7(4)$ \\
\hline C3 & $0.1151(5)$ & $0.5286(9)$ & $0.9981(3)$ & $3.9(4)$ \\
\hline C6 & $0.2125(6)$ & $0.3087(11)$ & $0.8637(3)$ & $6.2(6)$ \\
\hline C7 & $0.2906(6)$ & $0.3985(11)$ & $0.8707(3)$ & $5.6(5)$ \\
\hline $\mathrm{C} 8$ & $0.3471(6)$ & $0.3634(11)$ & $0.9196(3)$ & $5.6(5)$ \\
\hline $\mathrm{C} 9$ & $0.3040(7)$ & $0.2540(10)$ & $0.9423(3)$ & $5.7(6)$ \\
\hline $\mathrm{C} 10$ & $0.2197(7)$ & $0.2189(10)$ & $0.9078(4)$ & $5.9(6)$ \\
\hline C11A & $0.3501(6)$ & $0.7647(10)$ & $0.9354(3)$ & $4.9(5)$ \\
\hline $\mathrm{C} 12 \mathrm{~A}$ & $0.3116(7)$ & $0.8256(15)$ & $0.8882(4)$ & $10.5(8)$ \\
\hline C13A & $0.3605(9)$ & $0.9125(16)$ & $0.8628(5)$ & $12.5(10)$ \\
\hline C14A & $0.4497(8)$ & $0.9377(14)$ & $0.8840(5)$ & $10.5(9)$ \\
\hline C15A & $0.4913(7)$ & $0.8842(16)$ & $0.9305(5)$ & $10.8(9)$ \\
\hline $\mathrm{C} 16 \mathrm{~A}$ & $0.4408(7)$ & $0.7983(13)$ & $0.9566(4)$ & $8.1(7)$ \\
\hline C11B & $0.3509(5)$ & $0.6268(10)$ & $1.0349(3)$ & $4.6(5)$ \\
\hline C12B & $0.3695(6)$ & $0.7473(11)$ & $1.0686(4)$ & $6.3(6)$ \\
\hline C13B & $0.4172(7)$ & $0.7279(13)$ & $1.1191(4)$ & $7.9(7)$ \\
\hline C14B & $0.4459(7)$ & $0.5916(14)$ & $1.1383(4)$ & $8.7(7)$ \\
\hline C15B & $0.4281(7)$ & $0.4727(13)$ & $1.1063(4)$ & $8.2(7)$ \\
\hline $\mathrm{C} 16 \mathrm{~B}$ & $0.3807(7)$ & $0.4923(11)$ & $1.0550(3)$ & $6.3(6)$ \\
\hline C21A & $0.0777(5)$ & $0.7091(9)$ & $0.8493(3)$ & $3.9(4)$ \\
\hline $\mathrm{C} 22 \mathrm{~A}$ & $0.0386(6)$ & $0.8433(10)$ & $0.8543(3)$ & $5.5(5)$ \\
\hline $\mathrm{C} 23 \mathrm{~A}$ & $0.0322(7)$ & $0.9510(11)$ & $0.8162(4)$ & $6.9(6)$ \\
\hline C24A & $0.0629(7)$ & $0.9208(11)$ & $0.7724(4)$ & $7.2(6)$ \\
\hline $\mathrm{C} 25 \mathrm{~A}$ & $0.1005(7)$ & $0.7883(11)$ & $0.7661(3)$ & $6.1(6)$ \\
\hline $\mathrm{C} 26 \mathrm{~A}$ & $0.1095(6)$ & $0.6817(10)$ & $0.8045(3)$ & $5.2(5)$ \\
\hline $\mathrm{C} 21 \mathrm{~B}$ & $-0.0152(5)$ & $0.4706(9)$ & $0.8833(3)$ & $3.7(4)$ \\
\hline C22B & $-0.0165(6)$ & $0.3223(9)$ & $0.8943(3)$ & $4.7(5)$ \\
\hline $\mathrm{C} 23 \mathrm{~B}$ & $-0.1001(7)$ & $0.2473(11)$ & $0.8855(4)$ & $6.4(6)$ \\
\hline C24B & $-0.1821(6)$ & $0.3194(13)$ & $0.8651(4)$ & $7.0(7)$ \\
\hline C25B & $-0.1798(6)$ & $0.4668(13)$ & $0.8556(4)$ & $7.1(7)$ \\
\hline $\mathrm{C} 26 \mathrm{~B}$ & $-0.0970(6)$ & $0.5426(11)$ & $0.8634(3)$ & $5.4(5)$ \\
\hline C & $0.3251(10)$ & $0.1428(24)$ & $0.2110(5)$ & $19.7(16)$ \\
\hline $\mathrm{Cll}$ & $0.3485(4)$ & $-0.0461(6)$ & $0.23772(17)$ & $18.7(4)$ \\
\hline $\mathrm{Cl} 2$ & $0.2164(4)$ & $0.1465(10)$ & $0.22710(18)$ & $27.9(7)$ \\
\hline $\mathrm{Cl} 3$ & $0.3893(5)$ & $0.2438(8)$ & $0.23430(25)$ & $36.6(7)$ \\
\hline $\mathrm{O}$ & $0.9722(13)$ & $0.9688(24)$ & $0.0341(6)$ & $33.4(21)$ \\
\hline
\end{tabular}

chemical shift of the complex of PNP, the ${ }^{31} \mathrm{P}$ shifts at 84.6 and $102.5 \mathrm{ppm}$ are assigned to the phosphino sites attached to the primary and secondary carbons, respectively, of the PPN ligand in the complex [( $P, P^{\prime}, N$-PPN $)$ $\mathrm{FeCp]I}$.

Formation of Imino Complexes. When a chloroform solution of compound 10 was exposed to air, the corresponding imine complex 15 was formed quantitatively. The amine complex 12 was unstable in the presence of air; it converted immediately into the imine complex 16. In contrast to these two complexes, the other amine complexes 11, 13, and 14 resisted formation of iminoiron complexes.
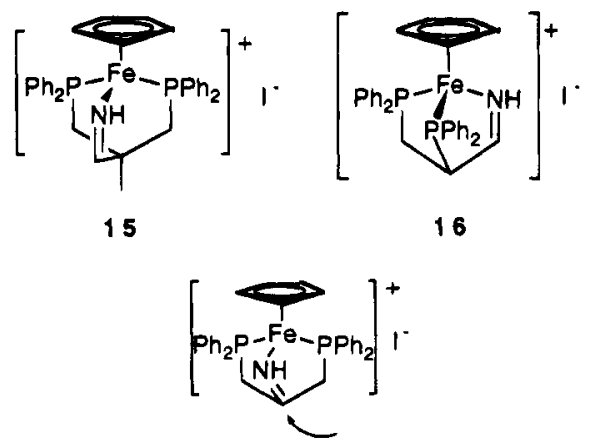
Table 5. 31P NMR Spectral Data for Complexes 11-19

\begin{tabular}{|c|c|}
\hline complex & ${ }^{31} \mathrm{P}$ NMR, $\delta[\Delta \delta]^{a}$ \\
\hline $\begin{array}{l}{\left[\left(P, P^{\prime}, N-\mathbf{P}_{2} \mathbf{N}\right) \mathrm{FeCp}\right] \mathrm{I}(\mathbf{1 0})} \\
{\left[\left(P, P^{\prime}, N-\mathrm{PNP}\right) \mathrm{FeCp}\right] \mathrm{I}(\mathbf{1 1})} \\
{\left[\left(P, P^{\prime}, N-\mathrm{PPN}\right) \mathrm{FeCp}\right] \mathrm{I}(\mathbf{1 2})} \\
{[(P, N-\mathbf{P N}-\mathbf{2}) \mathrm{FeCp}(\mathrm{CO})] \mathrm{I}(\mathbf{1 3})} \\
{[(P, N-\mathbf{P N}-3) \mathrm{FeCp}(\mathrm{CO})] \mathrm{I}(\mathbf{1 4})} \\
\mathbf{1 5} \\
\mathbf{1 6} \\
\mathbf{1 7} \\
\mathbf{1 8} \\
\mathbf{1 9}\end{array}$ & $\begin{array}{l}64.3[89.4] \\
77.7[100.1] \\
102.5(\mathrm{~d}, J=26 \mathrm{~Hz})[109.7] ; 84.6(\mathrm{~d}, J=26 \mathrm{~Hz})[104.6] \\
83.0[104.4] \\
55.5[80.6] \\
68.8[93.9] \\
137.5(\mathrm{~d}, J=20 \mathrm{~Hz})[144.7] ; 82.1(\mathrm{~d}, J=20 \mathrm{~Hz})[102.1] \\
60.2[85.3] \\
57.8(\mathrm{~d}, J=53 \mathrm{~Hz})[82.9] ; 19.4(\mathrm{~d}, J=53 \mathrm{~Hz})[79.4] \\
63.0(\mathrm{~d}, J=53 \mathrm{~Hz})[88.1] ; 24.3(\mathrm{~d}, J=53 \mathrm{~Hz})[84.3]\end{array}$ \\
\hline
\end{tabular}

${ }^{a}$ The numbers in brackets are coordination chemical shifts $\Delta \delta$ in ppm: $\Delta \delta=\delta$ (complex) $-\delta$ (free phosphine).

The oxidation process consists of initial oxidation of the metal center followed by oxidation of the coordinated amines to produce the imino moiety. ${ }^{15}$ The small halfwave potential of $11\left(\mathrm{PF}_{6}{ }^{-}\right.$salt $)$in acetonitrile $\left(E_{1 / 2}=\right.$ $0.69 \mathrm{~V} \mathrm{vs} \mathrm{Ag} / \mathrm{AgCl}$ ) and the similar ligand environments about the metal center in 11 and 12 (two phosphorus and one nitrogen donor) lead us to expect the complex to convert into an imino complex. As this did not occur, we sought to explain why the an imino complex was not formed. We speculate that such a reaction in 11 would result in a $\mathrm{C}=\mathrm{N}$ bond at the position of a bridgehead in the bicyclic small ring system $\mathbf{I}$, which is energetically unfavorable. ${ }^{16}$

As for complexes 13 and 14, it appears that a carbonyl ligand at the metal center prevents the oxidation. A carbonyl ligand is a good $\pi$-acceptor ligand which allows a back-bonding interaction with the metal center. Such a back-bonding interaction generally increases the oxidation potential of metal ions. Indeed, the measurement of $E_{1 / 2}$ for $13\left(\mathrm{PF}_{6}{ }^{-}\right.$salt; $\left.1.12 \mathrm{~V}\right)$ and $14(1.08 \mathrm{~V}$; $\mathrm{PF}_{6}{ }^{-}$salt) vs $\mathrm{Ag} / \mathrm{AgCl}$ in acetonitrile gives values similar to that reported for $\left[\mathrm{CpFe}\left(\mathrm{PMe}_{3}\right)_{2}(\mathrm{CO})\right]^{+}(1.30 \mathrm{~V}),{ }^{17}$ showing that these complexes have larger oxidation potentials that inhibit the oxidation process from occurring. In order to demonstrate this argument, complex 18 was prepared by treatment of 14 with trimeth-

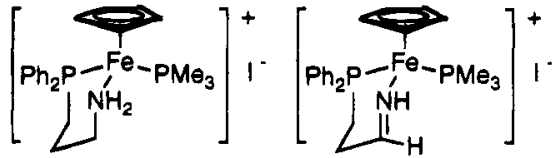

$$
\begin{aligned}
& 18 \quad 19
\end{aligned}
$$

ylphosphine. Complex 18 was slowly converted into the corresponding imine complex 19, as expected. Unfortunately, conversion of the iodide salt of 18 into a hexafluorophosphate or tetrafluoroborate resulted in the formation of its imino complex immediately, which caused difficulty in the measurement of the $E_{1 / 2}$ value for complex 18.

All of the imino complexes 15,16 , and 19 have been characterized by spectral and/or single-crystal analyses. The downfield chemical shifts at $\delta 8.43$ for $15, \delta 7.69$ for 16, and $\delta 8.69$ for 19 of $-\mathrm{HC}=\mathrm{NH}$ in the ${ }^{1} \mathrm{H}$ NMR spectra and $\delta 181.3\left(\mathrm{t}, J_{\mathrm{P}-\mathrm{C}}=5 \mathrm{~Hz}\right)$ for $15, \delta 173.3(\mathrm{t}$, $\left.J_{\mathrm{P}-\mathrm{C}}=4.7 \mathrm{~Hz}\right)$ for 16 , and $\delta 181.1\left(\mathrm{dd},{ }^{3} J_{\mathrm{P}-\mathrm{C}}=8,3 \mathrm{~Hz}\right.$ ), for 19 of $-\mathrm{HC}=\mathrm{NH}$ in the ${ }^{13} \mathrm{C}$ NMR spectra clearly indiate the existence of the imine functionality.

(15) Brown, G. M.; Weaver, T. R.; Keene, F. R.; Meyer, T. J. Inorg. Chem. 1976, 15, 190.

(16) Shea, K. J. Tetrahedron 1980, 36, 1683

(17) Treichel, P. M.; Komar, D. A. J. Organomet. Chem. 1981, 206 ,

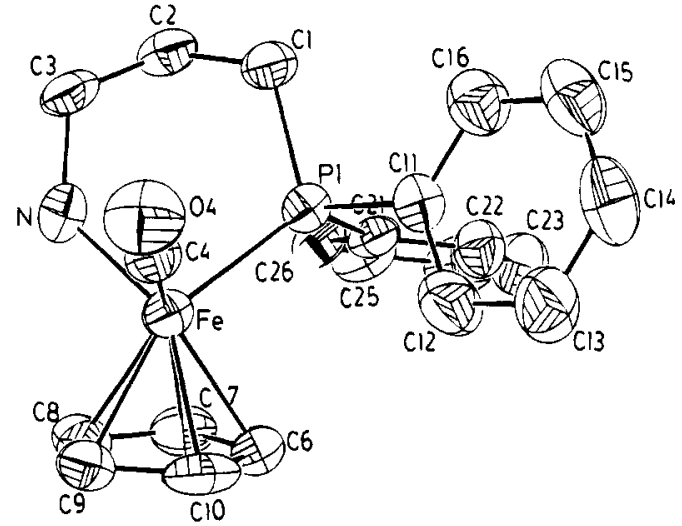

Figure 1. ORTEP plot of $[\mathrm{CpFe}(\mathrm{CO})(P, N-\mathrm{PN}-3)]^{+}$, the cation of 14 .

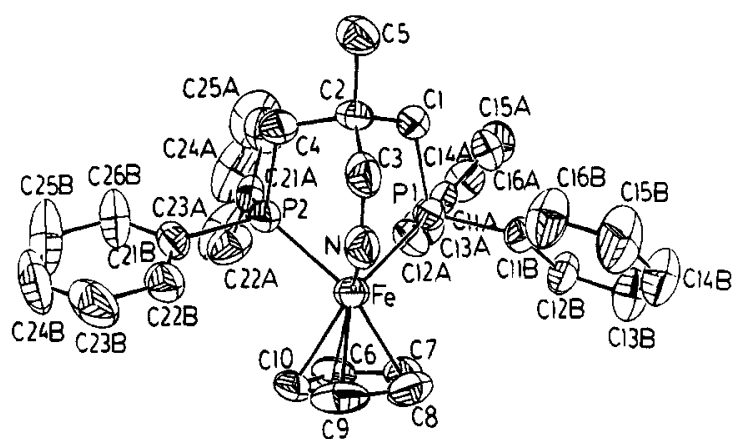

Figure 2. Crystal structure of the cation of complex $\mathbf{1 5 .}$

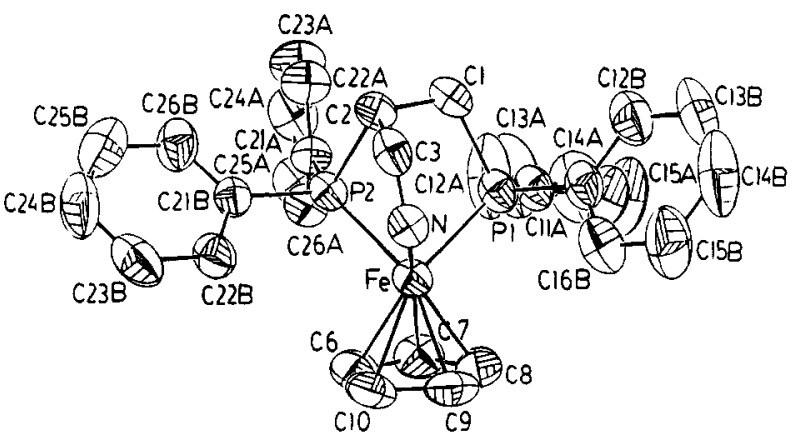

Figure 3. Crystal structure of the cation of complex 16.

Discussion of Crystal Structures. Single crystals suitable for X-ray analysis of complexes $14 \cdot \mathrm{CHCl}_{3}$, $15 \cdot \mathrm{H}_{2} \mathrm{O} \cdot \mathrm{CHCl}_{3}$ and $16 \cdot \mathrm{H}_{2} \mathrm{O} \cdot \mathrm{CHCl}_{3}$ were obtained in each case by slow evaporation of a chloroform/pentane solution in air. The crystal data for these complexes are summarized in Table 1, and atomic coordinates for all three structures are given in Tables 2-4. ORTEP drawings of 14-16 are depicted in Figures 1-3. In all instances, the iron atom displays a slightly distorted 
Table 6. Selected Bond Distances $(\AA)$ and Angles (deg)

\begin{tabular}{lccc}
\hline & $\mathbf{1 4}(\mathrm{X}=\mathrm{C} 4)$ & $\mathbf{1 5}(\mathrm{X}=\mathrm{P} 2)$ & $\mathbf{1 6}(\mathrm{X}=\mathrm{P} 2)$ \\
\hline $\mathrm{Fe}-\mathrm{P} 1$ & $2.189(2)$ & $2.182(3)$ & $2.199(3)$ \\
$\mathrm{Fe}-\mathrm{X}$ & $1.721(7)$ & $2.194(3)$ & $2.186(3)$ \\
$\mathrm{Fe}-\mathrm{N}$ & $2.027(5)$ & $1.911(8)$ & $1.928(6)$ \\
$\mathrm{N}-\mathrm{C} 3$ & $1.493(9)$ & $1.31(1)$ & $1.26(1)$ \\
$\mathrm{Fe}-\mathrm{C} 6$ & $2.070(7)$ & $2.10(1)$ & $2.079(9)$ \\
$\mathrm{Fe}-\mathrm{C} 7$ & $2.112(7)$ & $2.07(1)$ & $2.076(8)$ \\
$\mathrm{Fe}-\mathrm{C} 8$ & $2.120(7)$ & $2.10(1)$ & $2.074(8)$ \\
$\mathrm{Fe}-\mathrm{C} 9$ & $2.096(7)$ & $2.09(1)$ & $2.094(9)$ \\
$\mathrm{Fe}-\mathrm{C} 10$ & $2.059(7)$ & $2.068(9)$ & $2.111(9)$ \\
$\mathrm{P} 1-\mathrm{Fe}-\mathrm{X}$ & $90.4(2)$ & $91.6(1)$ & $87.2(1)$ \\
$\mathrm{P} 1-\mathrm{Fe}-\mathrm{N}$ & $94.8(2)$ & $86.3(2)$ & $83.5(2)$ \\
$\mathrm{X}-\mathrm{Fe}-\mathrm{N}$ & $94.7(3)$ & $87.9(3)$ & $79.6(2)$
\end{tabular}

octahedral geometry with one face occupied by the cyclopentadienyl moiety. All $\mathrm{Fe}-\mathrm{P}$ bond lengths in the range of 2.199-2.182 $\AA$ lie within the normal range (Table 6), but the distances of iron to nitrogen vary from amino to imino complexes; the average $\mathrm{Fe}-\mathrm{N}$ distance $(1.92 \AA)$ of imino complexes is slightly smaller than that for the amino complex $14(2.02 \AA)$ by about $0.1 \AA$, indicating that the imine functionality has a stronger coordinating ability toward the iron center than the amine one. As for the $\mathrm{N}-\mathrm{C} 3$ bond distance, the length $1.493(9) \AA$ in 14 is characteristic of a $\mathrm{C}-\mathrm{N}$ single-bond distance, whereas the lengths $1.31(1) \AA$ for 15 and 1.26(1) $\AA$ for $\mathbf{1 6}$ are in the typical range of a $\mathrm{C}=\mathrm{N}$ bond, which is consistent with spectral data. The chelate ring of $\mathrm{Fe}-\mathrm{P}-\mathrm{C} 1-\mathrm{C} 2-\mathrm{C} 3-\mathrm{N}$ in 14 adopts a stable chair form, as is evident from the examination of the torsional angles around this ring $\left(\mathrm{Fe}-\mathrm{N}-\mathrm{C} 3-\mathrm{C} 2,63.3(4)^{\circ}\right.$; $\mathrm{N}-\mathrm{C} 3-\mathrm{C} 2-\mathrm{C} 1,-74.9(6)^{\circ}$; $\mathrm{C} 3-\mathrm{C} 2-\mathrm{C} 1-\mathrm{P} 1,65.0(5)^{\circ}$; $\mathrm{C} 2-\mathrm{C} 1-\mathrm{P} 1-\mathrm{Fe},-41.8(3)^{\circ} ; \mathrm{C} 1-\mathrm{P} 1-\mathrm{Fe}-\mathrm{N}, 24.9(3)^{\circ}$; $\left.\mathrm{P} 1-\mathrm{Fe}-\mathrm{N}-\mathrm{C} 3,-37.3(3)^{\circ}\right)$; the typical alternation between positive and negative values is characteristic of a chair conformation.

\section{Summary}

The results reported here provide further examples of iron(II) amine complexes resistant to formation of their imino-type species. The iron metal center possessing a $\pi$-acceptor ligand has an increased oxidation potential, which prevents oxidation of the coordinated amine moiety. Once the carbonyl ligand is replaced by a $\sigma$-donor ligand such as phosphine, the oxidation process for the formation of imine moiety becomes feasible. Formation of an energetically unfavorable $\mathrm{C}=\mathrm{N}$ bond would also suppress oxidation.

Acknowledgment. We are grateful for the financial support of the National Science Council of the Republic of China (Grant No. NSC83-0208-M-002-63).

Supplementary Material Available: Tables giving anisotropic thermal parameters and all bond distances and angles for 14-16 (9 pages). Ordering information is given on any current masthead page.

OM940299S 